\section{Autonomy and accountability}

A

s news and features editor Patrick Sullivan points out in his preview of the CMA's third annual leadership conference, to be held Mar. 1 and 2 (see page 341 ), the topics of autonomy and accountability in health care promise to provoke spirited debate among the conference participants. On the one side are those who would, I sometimes think, turn back the clock to the days when physicians were apparently free to practise how and where they liked and to set their own price on the services they delivered. On the other side are those who believe that physicians have reached the limits of their ability to improve the health care of Canadians and that further improvements will arise not from advances in medical knowledge and technology but, rather, from broad social and economic changes.

When the debate heats up, innocent observers might be misled to believe that Canadian patients are dropping like flies for lack of appropriate health care and that governments are scrutinizing physicians' every move with an intensity exceeding the worst horrors of an orwellian nightmare.

We must remember that Canada's health care system, albeit imperfect, has served Canadians - and Canadian physicians well. We must also remember, however, that the health care needs and demands of Canadians are changing and will continue to challenge our ability to provide accessible and affordable care. To respond to these changes responsibly physicians, other health professionals, governments, institutions and the public must continue to discuss their needs, aspirations and concerns openly and cooperatively.

The CMA's leadership conference should provide such a forum. Through it we hope that Canada will take one additional step, however small, toward ensuring that its health care system will maintain the right of all $\mathrm{Ca}$ nadians to receive exemplary and accessible health care.

\section{Autonomie et responsabilité}

$\mathrm{P}$ atrick Sullivan, rédacteur aux informations générales, souligne dans son survol de la troisième Conférence annuelle de l'AMC sur le leadership, prévue pour les $1^{\text {er }}$ et 2 mars (voir page 341), que l'autonomie et la responsabilité dans le domaine des soins de santé soulèveront un débat animé parmi les participants à la conférence. Selon moi, il y a ceux qui aimeraient retourner aux pratiques d'antan où les médecins pouvaient apparemment s'établir où ils le voulaient, soigner à leur guise et fixer leurs honoraires comme ils l'entendaient. À l'inverse, d'autres croient que les médecins ne peuvent plus améliorer les services de santé destinés à la population canadienne et que toute évolution résultera de changements sociaux et économiques majeurs plutôt que de l'avancement des connais- sances et des technologies médicales.

Lorsque le débat atteindra son paroxysme, les observateurs dans les coulisses pourraient être portés à croire que les malades tombent comme des mouches faute de soins adéquats et que les médecins font l'objet, de la part des gouvernements, d'une surveillance serrée qui dépasse les pires horreurs dignes d'un cauchemar orwellien.

Nous ne devons pas oublier que le système de soins de santé du Canada, quoique imparfait, a bien servi la population canadienne - et les médecins canadiens. Nous devons toutefois reconnaitre que les besoins de notre population en matière de soins de santé changent et qu'ils continueront de poser un défi à notre capacité de prodiguer des soins accessibles à prix raisonnable. Les médecins, les autres professionnels de la santé, les gouvernements, les établissements et le public doivent continuer d'entretenir un dialogue ouvert sur leurs besoins, leurs aspirations et leurs inquiétudes dans un esprit de collaboration afin de répondre aux changements de façon responsable.

La conférence de l'AMC sur le leadership devrait fournir un tel forum et, nous l'espérons, permettre au Canada de faire ne serait-ce qu'un petit pas en avant pour s'assurer que son système de soins de santé maintienne le droit de tous les Canadiens à des services de santé exemplaires et accessibles.

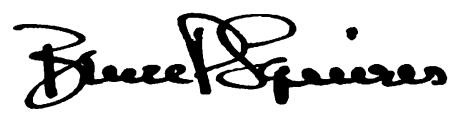

Bruce P. Squires, MD, PhD

Editor-in-chief

Rédacteur en chef 